\section{The bird brained and beyond}

\author{
Helmut V.B. Hirsch
}

Memory, Imprinting, and the Brain: An Inquiry into Mechanisms. By Gabriel Horn. Clarendon: 1986. Pp.315. Hbk £25, $\$ 44 ; p b k £ 15, \$ 24.95$.

HERE is a highly personal chronicle of the efforts of one group of investigators to understand information storage in the brain. First comes localization of the sites at which information is stored, then the attempt to understand the function of these sites and their relationship to other parts of the brain. The ultimate goal is to study the cellular mechanisms of information storage. Twelve chapters describe this approach, taking the reader step by step through a series of studies on habituation and on imprinting in birds. An appendix giving appropriate details is provided for those unfamiliar with bird neuroanatomy.

The author demonstrates a quality rare in today's world of scientific specialization. He has set himself the task of studying a very important biological problem without allowing himself to be boxed in by the methods at his command, or the borders of recognized fields. Where necessary he sought the help of others and has consistently designed interesting and informative experiments. No one experiment is "critical", but a progressive series of them leads us quite a way towards understanding information storage in the nervous system.

We see in this book how a research programme can grow and develop along with techniques in the field. In early experiments, labelled amino acids were used to localize crudely areas in the chick brain in which protein synthesis, and thus possibly activity and growth, is high during imprinting. Subsequently, autoradiography was used to home in on the exact location of areas involved in information storage. Thus, technical advances coupled with experimental ingenuity were crucial in advancing understanding of imprinting and of the underlying process of information storage.

The first, and perhaps the weakest, chapter of the book deals with the decrement in neuronal and behavioural responses upon repeated presentation of the same stimulus. Habituation is compared with other forms of learning that involve elaboration of a new response or modification (not simply loss) of an old one. Imprinting in birds is put forth as a model for studying these forms of information storage; this is the focus of the remainder of the book.

Incorporation of radioactive lysine or radioactive uracil is used to identify brain regions at which there are high levels of activity and/or growth during imprinting. One hypothesis is that these are potential sites for information storage during imprinting, but there are alternative hypotheses. For example, incorporation of lysine or uracil at these sites may reflect nonspecific side-effects of training, such as differences in activity level between imprinted and control animals. By combining careful behavioural observations with studies of incorporation of uracil, Horn has been able to rule out many of the alternative hypotheses.

Autoradiographic techniques increase the precision in identifying potential sites for information storage during imprinting; a restricted part of the forebrain, the "intermediate and medial part of the hyperstriatum ventrale" (IMHV), can be identified with this procedure. The author would, however, probably be the first to admit that in order to test critically the hypothesis that the IMHV is the area that stores information during imprinting, manipulative neural experiments must be performed.

Some aspects of the imprinting process involve areas outside the IMHV. Chicks will imprint on many different types of target, but they appear to have a predisposition to prefer certain "biologically appropriate" objects such as a stuffed, rotating jungle fowl. This predisposition is probably determined intrinsically, because it does not require previous exposure to the appropriate target, but can be activated by exposure to a lighted environment or running in an activity wheel in the dark; it is not abolished by lesioning the IMHV.

There is further evidence that neural systems concerned with preferences for biologically appropriate imprinting targets are to some extent distinct from those concerned with storage of the information needed to identify particular objects as familiar. A reduction in the level of noradrenaline in the brain affects imprinting to targets such as a rotating, flashing red box, but not to the jungle fowl. In contrast, testosterone levels in the plasma are correlated with imprinting to the jungle fowl, but not to the red box. A preference for the jungle fowl may therefore reflect a special-purpose information storage system, one affected by testosterone levels; learning to recognize a particular object as familiar may involve a second, generalpurpose mechanism, one modulated by the level of noradrenaline. Under natural conditions imprinting probably takes place at an age when the predisposition for natural stimuli is fully developed and ensures that the chick will select an appropriate imprinting target. Continued exposure to a particular target allows the chick to store information needed to recognize it as familiar. Thus the chick can distinguish its mother from other females of the species.

There are some flaws in the book, mostly cosmetic. There are far too many typographical errors; in several places parts of sentences are missing. Also, figure 5.3 is mislabelled, and several figures lack error bars. It is a great shame that minor irritants such as these were allowed to intrude into what is otherwise a most impressive book.

Horn's monograph speaks to several audiences. It gives students of animal behaviour an impetus to consider the neuronal basis of behaviour, and it shows neuroscientists the power of carefully designed behavioural studies. Indeed, the book is a reminder that a very basic goal in neuroscience is understanding the role of the nervous system in behaviour. Finally, and perhaps most important, in the same fine tradition as K.D. Roeder's Nerve Cells and Insect Behavior it will inspire students to take up the challenge posed by the nervous system. The author has prepared a vade-mecum; taking us step by step along his path, he presents a highly personal account - but without blowing his own horn.

Helmut V.B. Hirsch is Professor in the Neurobiology Research Center, State University of New York at Albany, Albany, New York 12222, USA.

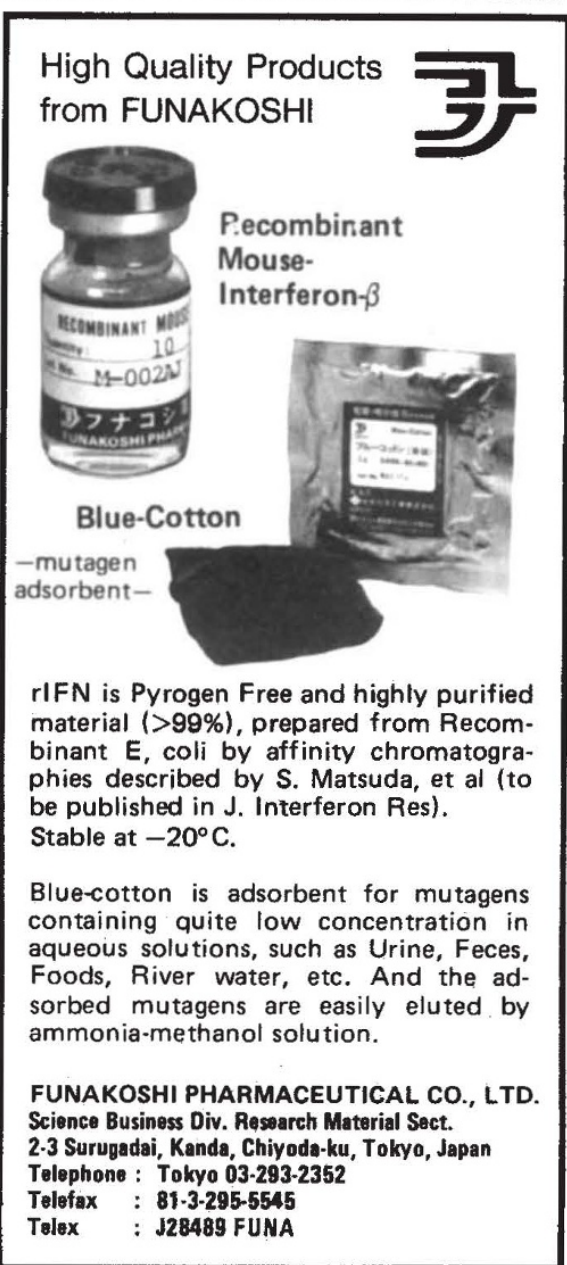

Reader Service No.21 